\title{
Artificial Intelligence Algorithm with SVM Classification using Dermascopic Images for Melanoma Diagnosis
}

\section{Vivekanadam Balasubramaniam,}

Faculty of Computer Science and Multimedia,

Lincoln University College,

Kota Bharu, Malaysia.

vivekanandam@lincoln.edu.my

\begin{abstract}
Of all suspicious pigmented skin lesions considered for analysis, a large portion is often benign. The pressure of pathology services and secondary care must be reduced throughout the patient trials using modern techniques for improving the melanoma diagnosis accuracy. Dermoscopic images obtained from digital single-lens reflex (DSLR) cameras, smartphones and a lightweight USB camera are compared using artificial intelligence (AI) algorithm for determining the accuracy of melanoma identification. Datasets are obtained from thousand test samples undergoing plastic surgery. The diagnostic trial is masked, single arm and multicentered. The controlled and suspicious skin lesions as well as the suspicious pigmented skin lesion are captured on the aforementioned cameras while scheduling for biopsy. The possibility of melanoma is assessed using deep learning (DL) techniques on the pigmented skin lesions seen in the dermascopic images for identifying melanoma. For this purpose, we train a deterministic AI algorithm based on malignancy recognition by deep ensemble and inputs from clinicians. The histopathology diagnosis is used as a standard criterion for determining the specialist assessment, algorithmic specificity, sensitivity and the area under the receiver operating characteristic curve (AUROC).
\end{abstract}

Keywords: Artificial Intelligence, Deep Learning, Melanoma, Image Processing, Medical Imaging

\section{Introduction}

Malignant melanoma is a comparatively rare form of skin cancer that is increasing rapidly over the past decade. It has been found to be one of the major causes of death by skin cancer [1]. A relative survival rate of 5-years can be attained for $95 \%$ of the patients who are diagnosed 
Journal of Artificial Intelligence and Capsule Networks (2021)

Vol.03/ No.01

Pages: $34-42$

http://irojournals.com/aicn/

DOI: https://doi.org/10.36548/jaicn.2021.1.003

with stage 1 melanoma. However, when the disease is diagnosed at stage 4, this survival rate reduces to 8 to $25 \%$. Histopathology assessment, excision, specialist assessment, primary care physician evaluation and self-evaluation steps are undertaken before the definitive melanoma diagnosis by an individual with a suspicious pigmented skin lesion [2]. Dermoscopy is to be used for assessment of any suspicious skin lesions by properly trained health care professionals according to the recommendation of the current practice guidelines.

The demarcation concerning potentially harmful and harmless lesions may be improved at each step of diagnosis to improve the accuracy of the techniques to reduce the unnecessary references of later services caused by the lesions [3]. A comprehensive series of reviews based on the accuracy of various diagnostic schemes inclusive of smartphone applications, computeraided diagnostic techniques, tele-dermatology, reflectance confocal microscopy, as well as visual assessment with or without dermoscopy are published recently by the Cochrane Skin Cancer Diagnostic Test Accuracy Group [4]. From this review, it is evident poor quality and limited data exists supporting the widespread adoption of smartphone applications, computer assisted diagnostic schemes and tele-dermatology [5]. When compared to just visual inspection, the diagnostic accuracy is improved by dermoscopy. When compared to only dermoscopy, the diagnostic accuracy is improved using reflectance confocal microscopy [6]. The reflectance confocal microscopy equipment are pricey. Due to this reason, they are seldom used and the experience of the examiners largely affects the accuracy of clinical diagnostics.

Despite minimal clinical validation evidences, melanoma detection based applications are released by several smartphone application developers [7]. The malignancy probability was estimated in 4 out of 50 smartphone photographs assessed for skin cancer. However, the accuracy of diagnostics has not been assessed. Patients may be affected by misleading, inaccurate and poorly designed consumer applications. The diagnostic accuracy can be largely improved by incorporation of modern electronic technology, appropriate evaluation and suitable development [8]. The AI based melanoma classification algorithms for categorizing photographs of lesions have reached the competence level that is comparable with the dermatologists in the recent days. Skin Analytics Limited developed an AI algorithm for malignancy recognition based on deep ensemble, used for analyzing dermoscopic images of pigmented skin lesions for identifying the likelihood of skin cancer by healthcare professionals for decision support [9]. Around 10000 dermoscopic images that are archived are compared for assessment and identification of melanoma related lesion features using deep learning 
Journal of Artificial Intelligence and Capsule Networks (2021)

Vol.03/ No.01

Pages: $34-42$

http://irojournals.com/aicn/

DOI: https://doi.org/10.36548/jaicn.2021.1.003

schemes. The results of analysis show that the accuracy of melanoma identification by this algorithm is comparable to that of specialist physicians [10].

\section{Proposed Scheme}

A masked, single-arm, multicenter prospective diagnostic trial is conducted. A dataset consisting of over 10,000 images of skin lesions is considered for the purpose of analysis. Images with biopsied or no skin lesions were withdrawn as ineligible. Details such as race, age and gender of the person is obtained from the database. Specialist opinion is also taken during selection of image samples. Five benign and control lesions of diameter less than $15 \mathrm{~mm}$ are considered that are not excised or biopsied previously. They are chosen such that they are not located in an area of visible scarring or in a site that is anatomically not suitable for photographing. Images obtained from different cameras namely digital single-lens reflex (DSLR) based, smartphones based and a lightweight USB camera based are used for the sake of comparison. The study is conducted with up to date operating systems. DermLite lenses are attached to all the cameras for dermoscopic clarity. An ordinal scale is used for assessing the likelihood of melanoma by clinicians on a scale of 1 to 4 where 4 indicates most likely and 1 indicates least likely.

Dysplastic nevi [11], melanoma and other skin diseases are categorized based on the histopathology results obtained from the biopsied excised lesions. In situ melanoma is also categorized under melanoma. The most probable diagnosis is seen when histopathological uncertainties are reported during the diagnosis. The regular standard of care is used for managing the patients and the algorithmic assessment outcomes are not informed to the patients or the clinicians. Until the recruitment process is accomplished, the images were electronically stored. Previously published dermoscopic images are used for training the algorithm prior to the study. During the second stage, the algorithm is trained with a subset of 250 images extracted from the sample data set. Of these images, 172 images represented control lesions, 53 images had nonmelanoma lesions and 25 of them had melanoma lesions. In order to avoid overfitting, in both test split and training, no single patient indicated the presence of lesions. After training the algorithm with images from different cameras, assessment of the images is also performed. The data set removes the flawed or blurred images and quality screening is also performed on the images. Further, the rest of the images in the database are assessed by the algorithm. 


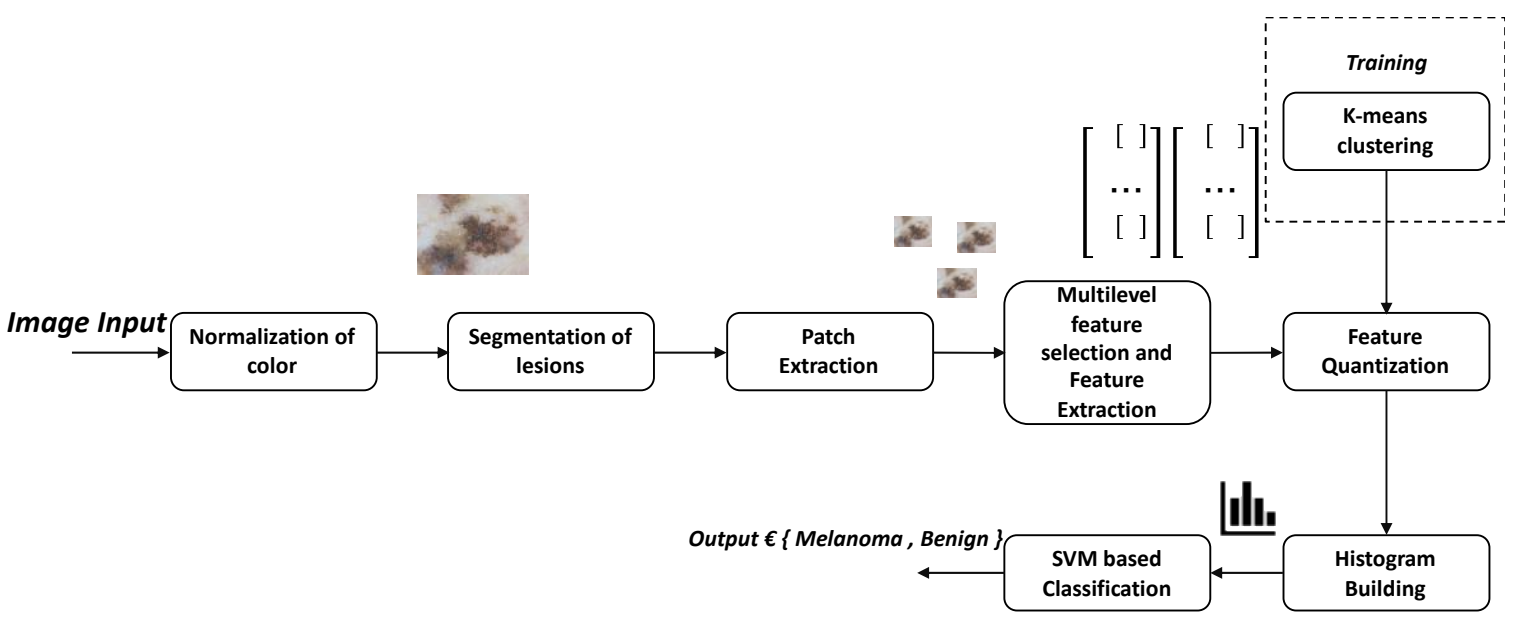

Figure 1. Proposed DL based Skin Cancer Detection Model

Figure 1 represents the proposed DL based skin cancer detection model where the input image is fed to the system where color normalization is performed. Further, the lesions are segmented and patch extraction is performed. Multilevel feature selection and feature extraction is performed. The system is trained using the k-means clustering algorithm and is combined with the extracted features for feature quantization. Further, histogram building [12] is performed and classification is performed using Support Vector Machine technique.

\section{Analysis}

For clinical assessment, each camera is evaluated for histopathology-confirmed diagnosis with criterion standard with the confidence scale of the algorithm. Uncertainty is calculated with bootstrapped estimation, a non-parametric technique with receiver operator curves. All biopsied lesions are estimated for the likelihood of melanoma based and the clinical accuracy of the assessment is compared. The lesions used for training of the algorithm and the ones whose images are missing are also included in this analysis. However, only lesions consisting of images from all three cameras are considered for analysis in direct comparison. False-negative rate, false positive rate, predictive value, specificity and other indices of diagnostic accuracy as well as the area under the receiver operating characteristic curve (AUROC) are compared for the possibility of melanoma at multiple decision thresholds generating sensitivities of $95 \%$ and $100 \%$.

Histopathology is used for assessment of all lesions considered for biopsy due to the utilization of $100 \%$ sensitivity threshold. Between the assessment, the AUROC equality is

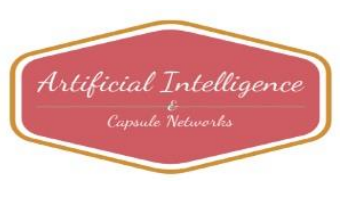


Journal of Artificial Intelligence and Capsule Networks (2021)

Vol.03/ No.01

Pages: $34-42$

http://irojournals.com/aicn/

DOI: https://doi.org/10.36548/jaicn.2021.1.003

tested using a $\chi^{2}$ test. The accuracy of various melanoma detection schemes are examined based on the influence of covariates. Location of lesion on specific region of the body, family or patient history of skin cancer or melanoma, hair color, Fitzpatrick skin type, race, sex, age and level of concern of the patient are some of the patient related covariates that are examined. Ransom tests are performed using the available data to assess the potential consequences of poor-quality images and other missing parameters. Selection model is used for estimating the modified receiver operating characteristic curve developed by Rajbhandari et. al. All tests are 2-tailed and statistical significance is attained at a $\mathrm{P}$ value less than 0.05. Stata statistical software (StataCorp) is used for performing the statistical analysis. An accuracy of over 96\% is obtained by the statistical estimates.

\section{Results and Discussion}

Fitzpatrick skin sensitivity is seen in most participants while no form of skin cancer existed in the personal or family history of such participants. The study population consisted of a mean age of 49 years with $62 \%$ female patients. During preprocessing, the images are prepared where unwanted elements of the image are eliminated so as to enable further processing. Moles, skin color, veins, hairs, low contrast and other artifacts are eliminated by the model. Small elements such as hairs in the image are detected using bottom hat filtering and are eliminated. Hair recognition and elimination can also be performed using the median filtered image for improving the image of skin lesion and further removal of noise. Grayscale conversion of the RGB image is also performed to retain only the intensity information from the image to enable quick image processing. SVM is a popular classifier that is applied on the extracted image features to classify the skin lesion into benign and melanoma. Control lesions and biopsied lesions are categorized as a result of analysis. It is further categorized into dysplastic nevi, and histopathology by melanoma.

Table 1: Comparison of Algorithm performance

\begin{tabular}{|l|c|c|c|c|c|c|c|}
\hline \multicolumn{1}{|c|}{ Technique } & NNB & NPV & PPV & FNR & FPR & Specificity & Sensitivity \\
\hline Clinician & 5.4 & 100 & 20 & 0 & 30 & 70 & 100 \\
\hline DSLR images & 10.2 & 100 & 9 & 0 & 70 & 30 & 100 \\
\hline Mobile Images & 7.5 & 100 & 13 & 0 & 49 & 50 & 100 \\
\hline $\begin{array}{l}\text { Lightweight USB camera } \\
\text { images }\end{array}$ & 5.1 & 100 & 18 & 0 & 35 & 65 & 100 \\
\hline
\end{tabular}

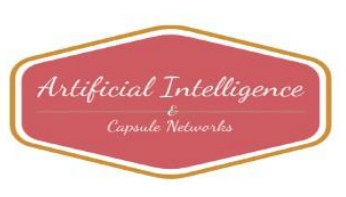


Journal of Artificial Intelligence and Capsule Networks (2021)

Vol.03/ No.01

Pages: $34-42$

http://irojournals.com/aicn/

DOI: https://doi.org/10.36548/jaicn.2021.1.003

Table 1 provides the comparison of the performance of the proposed algorithm on images obtained from the specific sources. NNB represents number needed for biopsy identification, NPV is the negative predictive value and PPV represents positive predictive value. FNR is false negative rate while false positive rate is represented by FPR. The skin lesion diameter, color, border and symmetrical nature are identified. The object's distributed intensity is obtained from texture analysis using gray level co-occurrence matrix (GLCM). A reference pixel and a neighboring pixel are considered for GLCM. Features inclusive of shade, prominence, homogeneity, entropy, energy, correlation and contrast are obtained by this technique.

(A) Clinician assessment

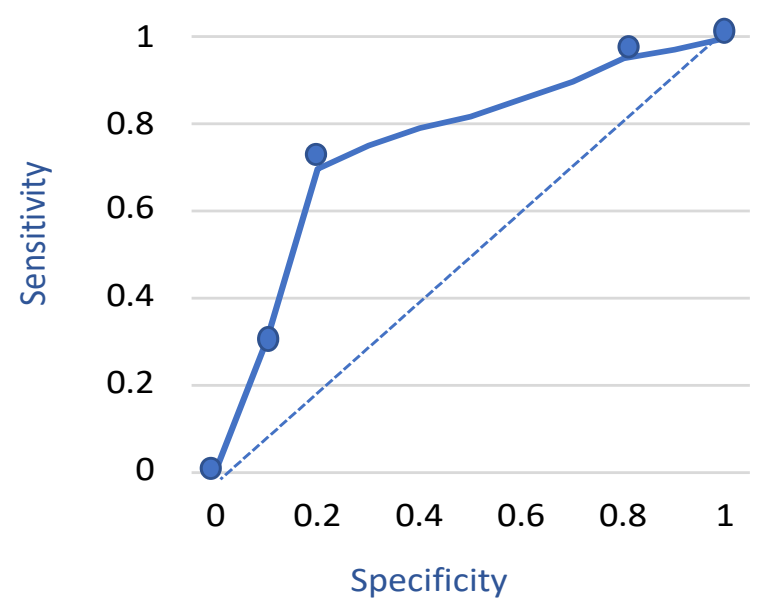

(C) Mobile images

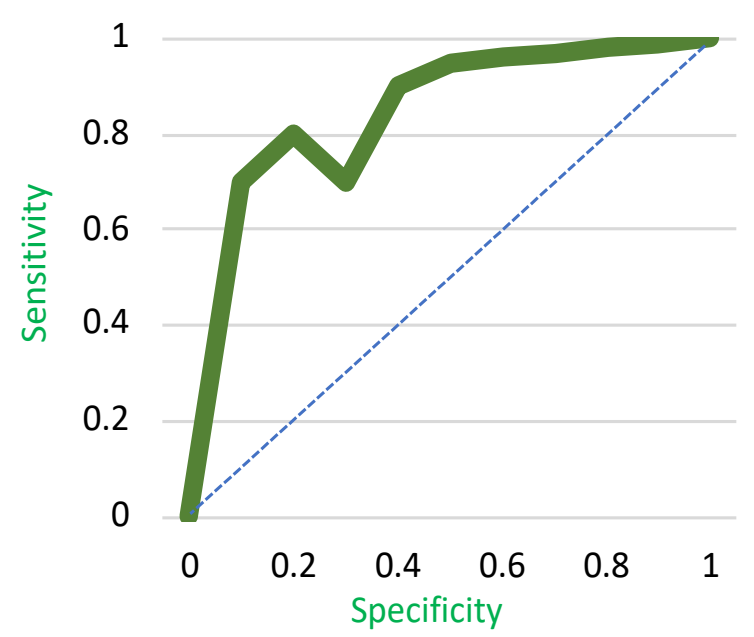

(B) DSLR Images

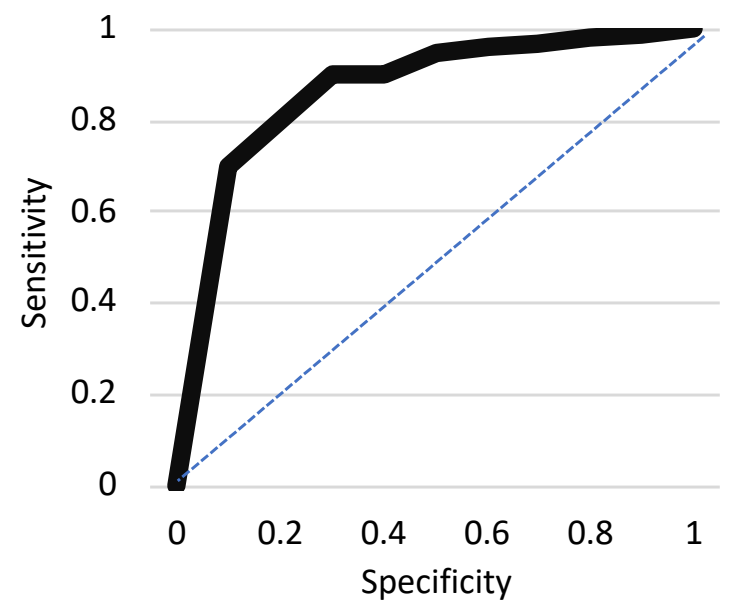

(D) Lightweight USB Camera images

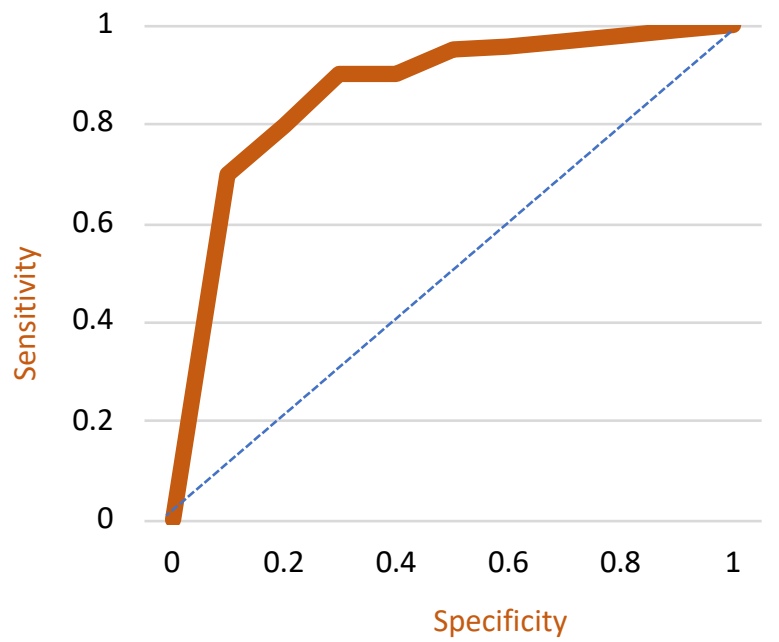

Figure 2: Area Under Curve of Receiver operator characteristics for (a) Clinician Assessment, (b) DSLR camera images, (c) Images obtained from mobile camera, and (d) Images obtained from the lightweight USB camera 
Journal of Artificial Intelligence and Capsule Networks (2021)

Vol.03/ No.01

Pages: $34-42$

http://irojournals.com/aicn/

DOI: https://doi.org/10.36548/jaicn.2021.1.003

Figure 2 provides the area under curve of receiver operator characteristics of the results obtained from testing the algorithm. Validation bias is observed during analysis of control lesions that did not contain biopsy. This lead to a decrease in specificity and increase in sensitivity. Physician's experience, risk of skin cancer, skin lesion type and frequency and patient population are some of the other significant factors that makes a prominent difference in the assessment. Lack of generalization may occur due to overfitting of AI systems. Imaging techniques, melanoma subtypes and patient demographics also significantly affects the outcomes of prediction. This dives the need for clinical validation of the obtained results. Minimal overfitting is observed by increasing the training iterations for the algorithm.

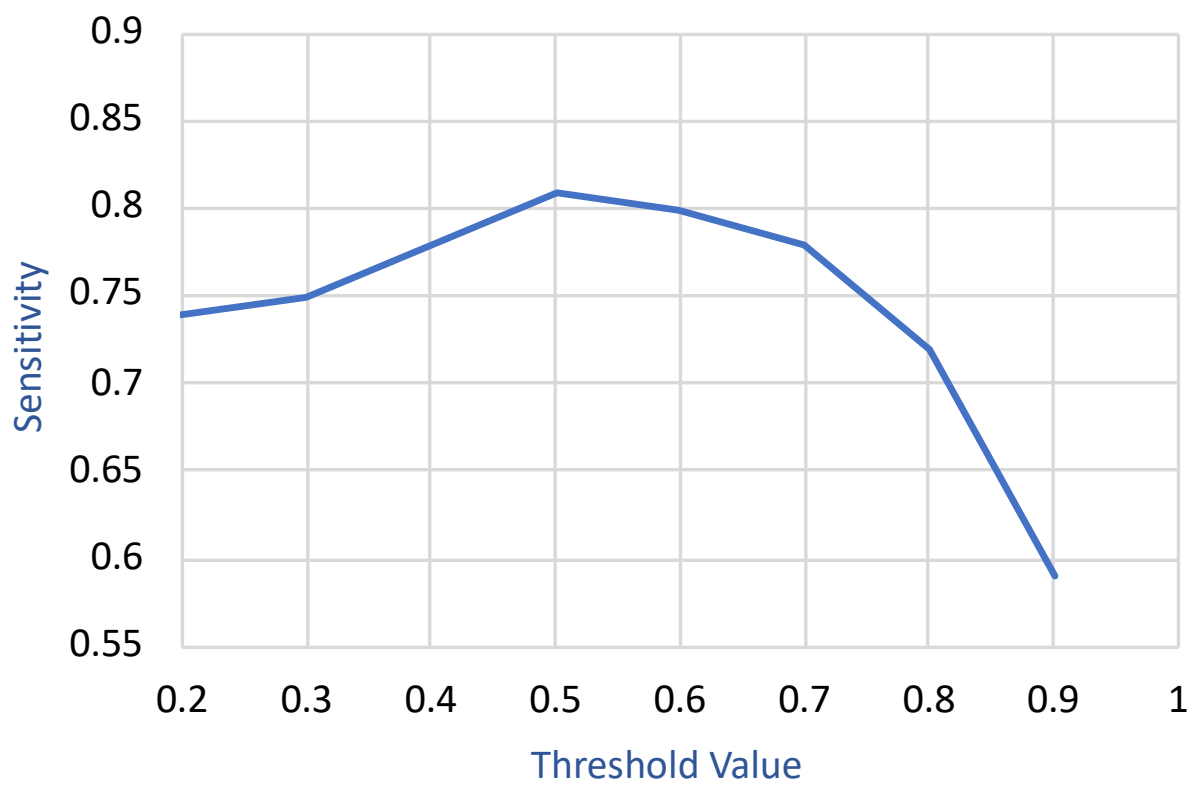

Figure 3: Sensitivity curve for varying threshold values

Figure 2 represents the sensitivity curve for varying threshold value. The pixels available in the identified skin area are equalized and the skin clusters are segmented based on the boundaries of the color space. Further, the run time performance of the algorithm is also assessed.

\section{Conclusion}

Detection of melanoma with an accuracy level similar to that of specialists is demonstrated by the proposed artificial intelligence algorithm with SVM classification. Dermoscopic images obtained from digital single-lens reflex (DSLR) cameras, smartphones

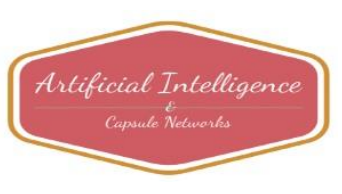


Journal of Artificial Intelligence and Capsule Networks (2021)

Vol.03/ No.01

Pages: $34-42$

http://irojournals.com/aicn/

DOI: https://doi.org/10.36548/jaicn.2021.1.003

and a lightweight USB camera are compared using artificial intelligence (AI) algorithm for determining the accuracy of melanoma identification. The dataset contained over 10,000 images of skin. The health care service efficiency is increased largely by transforming the patient diagnosis pathway using AI based services and low-cost screening techniques. The algorithm is trained with a subset of 250 images extracted from the sample data set. Of these images, 172 images represented control lesions, 53 images had nonmelanoma lesions and 25 of them had melanoma lesions. Minimal overfitting is observed by increasing the training iterations for the algorithm. Future work is directed towards inclusion of predictive diagnostic scheme for early cancer diagnostics based on pre-in situ technique. The melanoma classifiers accuracy can also be further enhanced to improve diagnosis.

\section{References}

[1] Sondermann, W., Utikal, J. S., Enk, A. H., Schadendorf, D., Klode, J., Hauschild, A., ... \& Brinker, T. J. (2019). Prediction of melanoma evolution in melanocytic nevi via artificial intelligence: A call for prospective data. European Journal of Cancer, 119, 3034.

[2] Pennisi, A., Bloisi, D. D., Nardi, D., Giampetruzzi, A. R., Mondino, C., \& Facchiano, A. (2016). Skin lesion image segmentation using Delaunay Triangulation for melanoma detection. Computerized Medical Imaging and Graphics, 52, 89-103.

[3] Tschandl, P., Codella, N., Akay, B. N., Argenziano, G., Braun, R. P., Cabo, H., ... \& Kittler, H. (2019). Comparison of the accuracy of human readers versus machinelearning algorithms for pigmented skin lesion classification: an open, web-based, international, diagnostic study. The Lancet Oncology, 20(7), 938-947.

[4] Adegun, A., \& Viriri, S. (2020). Deep learning techniques for skin lesion analysis and melanoma cancer detection: a survey of state-of-the-art. Artificial Intelligence Review, $1-31$.

[5] Codella, N., Rotemberg, V., Tschandl, P., Celebi, M. E., Dusza, S., Gutman, D., ... \& Halpern, A. (2019). Skin lesion analysis toward melanoma detection 2018: A challenge hosted by the international skin imaging collaboration (isic). arXiv preprint arXiv:1902.03368. 
Journal of Artificial Intelligence and Capsule Networks (2021)

Vol.03/ No.01

Pages: $34-42$

http://irojournals.com/aicn/

DOI: https://doi.org/10.36548/jaicn.2021.1.003

[6] OZKAN, I. A., \& KOKLU, M. (2017). Skin lesion classification using machine learning algorithms. International Journal of Intelligent Systems and Applications in Engineering, $5(4), 285-289$.

[7] Goyal, M., Knackstedt, T., Yan, S., \& Hassanpour, S. (2020). Artificial intelligencebased image classification for diagnosis of skin cancer: Challenges and opportunities. Computers in Biology and Medicine, 104065.

[8] Albahar, M. A. (2019). Skin lesion classification using convolutional neural network with novel regularizer. IEEE Access, 7, 38306-38313.

[9] Vijayakumar, T. (2019). Neural network analysis for tumor investigation and cancer prediction. Journal of Electronics, 1(02), 89-98.

[10] Bashar, A. (2019). Survey on evolving deep learning neural network architectures. Journal of Artificial Intelligence, 1(02), 73-82.

[11] Pandian, A. P. (2019). Identification and classification of cancer cells using capsule network with pathological images. Journal of Artificial Intelligence, 1(01), 37-44.

[12] Shakya, S. (2020). Analysis of artificial intelligence based image classification techniques. Journal of Innovative Image Processing (JIIP), 2(01), 44-54. 